\title{
Selectivity of oxidative stress targeting in estrogen-induced experimental nephrocarcinogenesis ${ }^{\star}$
}

\author{
Jarek Kobiela $^{1,2}$, Jacek Krajewski ${ }^{1,2}$, Beata Kalińska-Błach ${ }^{1}$ and Tomasz Stefaniak ${ }^{2 \bowtie}$ \\ ${ }^{1}$ Department of Medical Chemistry, Medical University of Gdańsk; ${ }^{2}$ Department of General, \\ Gastroenterological and Endocrinological Surgery, Medical University of Gdańsk, Gdańsk, \\ Poland
}

Received: 13 November, 2001; revised: 7 January, 2002; accepted: 7 February, 2002

Key words: oxidative stress, protein carbonyl group, renal carcinogenesis, estrogen-induced carcinogenesis

\begin{abstract}
Specificity of targeting of the oxidative stress towards lipid and protein fractions in a model of estrogen-induced Syrian hamster nephrocarcinogenesis was evaluated. The amount of proteins modified by oxidative stress was significantly elevated as early as one month after the initial implantation of estradiol to the experimental versus the control group, while the stress did not affect lipids. Subcellular localization of the oxidative stress target was determined by the analysis of protein oxidation in subcellular fractions of kidney cells. The endoplasmic reticulum membranes were the fraction most affected by the oxidative stress.
\end{abstract}

Estrogen dependent kidney cancer in male Syrian hamster was first described by Kirkman in the late fifties [1]. This model is restricted to male hamsters only $[1,2]$. Since the first report, the model has been thoroughly investigated in order to determine the molecular site of pathogenesis and histopathological origin of the cancer [2-5]. Although it has now become an accepted and very popular model of experimental tumorigenesis that is assumed to mimic hormone dependent hu- man cancer (e.g. of the breast, ovary or endometrium), there is still much to be explained [6-9].

It has been suggested that the cancer may be induced by oxygen free radical-mediated DNA damage in kidney cells. Liehr and coworkers [10, 11] and $\mathrm{Li} \& \mathrm{Li}$ [12] proposed oxidoreductive cycling between hydroxylated derivatives of estradiol and its semiquinone and quinone metabolic intermediates as a possible way of superoxide anion generation. On the

\footnotetext{
${ }^{\star}$ Presented at the Polish Japanese Free Radical Forum, 2001.

${ }^{\circledR}$ To whom correspondence should be addressed: Tomasz Stefaniak, Department of General, Gastroenterological and Endocrinological Surgery, Medical University of Gdańsk, Prof. Kieturakis 1, 80-742 Gdańsk, Poland; e-mail: wujstef@amedec.amg.gda.pl
}

Abbreviations: DNPH, 2,4-dinitrophenylhydrazine; 5-DSA, 5-doxylstearic acid; DTNB, 5,5'-dithio- bis(2nitrobenzoic acid). 
other hand, these authors also postulated that estrogen semiquinones could induce DNA adducts and, in this way, mutagenesis [10-12]. It has been suggested that hydroxylation in the position 2 or 4 , that leads to formation of catecholestrogens, may be a trigger point in the process of carcinogenesis [13, 14]. High activity of estradiol-4-hydroxylase has been observed in the organs susceptible to estrogen-induced carcinogenesis, i.e. in mouse uterus and male Syrian hamster kidney [15, 16] as well as in human breast cancer cells [17]. The catecholestrogen formed can be easily oxidised to semiquinone and quinone derivatives by cytochrome P450 [18, 19]. Zhu \& Conney [20] pointed to some properties of 4-hydroxyestradiol that might be of crucial importance for potential carcinogenicity, especially its ability to stimulate cell proliferation and growth, which was observed in cell lines of kidney epithelium [20-22].

The aim of this study was to investigate which of the subcellular fractions of the chronically estrogenized kidney tissue is most affected by oxidative stress and, moreover, which biomolecules, lipid or proteins, are most susceptible to this stress.

\section{MATERIALS AND METHODS}

Chemicals. Cumene hydroperoxide, 2,4-dinitrophenylhydrazine (DNPH) and guanidine-HCl were purchased from Sigma Chemical Co. (St. Louis, MO, U.S.A.). All other reagents were of the highest grade commercially available.

Thirty male Syrian hamsters aged 4 weeks were divided into two groups: 15 hamsters (group 1) were implanted subcutaneously with $25 \mathrm{mg}$ of $17-\beta$-estradiol (Sigma-Aldrich Co.). The other 15 animals (group 2) did not receive any treatment. After one-month experiment the animals were killed and their kidneys were excised for biochemical assays. The mass of testes was taken as a marker of estrogenization effectivity and proved to be at least 12 times lower in each of the animals treated with the estrogen as compared to the control.

All the animals were kept in the animal facility at room temperature, standard humidity and $12 \mathrm{~h}$ day/night circadian cycle. They were fed with standard chow and given water $a d l i$ bitum.

All the procedures concerning animals were approved by Local Ethical Committee (LEC) and performed according to the instructions authorized by LEC.

Preparation of homogenates. The kidneys from 5 animals of each group were combined and homogenized in $150 \mathrm{mM} \mathrm{KCl}, 10 \mathrm{mM}$ Tris/ $\mathrm{HCl}, \mathrm{pH} 7.4$, to form a $10 \%$ (for sufhydryl, carbonyl and lipid hydroperoxide assessments) or $20 \%$ (for subcellular fractions isolation) suspension.

Isolation of subcellular fractions. A $20 \%$ homogenate was prepared from excised kidneys in $150 \mathrm{mM} \mathrm{KCl}, 10 \mathrm{mM}$ Tris/HCl, $\mathrm{pH}$ 7.4, in a Potter-Elvehjem Teflon homogenizer by 50 gentle up-and-down strokes. Nuclei and cell debris were removed by centrifugation $(600 \times$ g, $10 \mathrm{~min}$ ), the supernatant was collected and centrifuged again $(10000 \times \mathbf{g}, 90 \mathrm{~s})$. The pellet (mitochondrial fraction) and supernatant were separated. The supernatant was recentrifuged $(15000 \times \mathbf{g}, 30 \mathrm{~min})$ in order to remove the light mitochondrial fraction. The supernatant obtained (cytosolic fraction) was centrifuged $60 \mathrm{~min}$ at $100000 \times \mathrm{g}$ in order to obtain the microsomal fraction.

Carbonyl group assessment. This was performed as described by Oliver et al. [23]. Each sample to be measured was prepared by combining $100 \mu \mathrm{l}$ of the homogenate with $100 \mu \mathrm{l}$ of DNPH (Sigma-Aldrich Co.), whereas the respective control sample was made of the equivalent amount of the homogenate and $100 \mu \mathrm{l} 2 \mathrm{M} \mathrm{HCl}$ (Sigma-Aldrich Co.). All the samples were incubated at room temperature for $60 \mathrm{~min}$ and vigorously shaken every 10 $\min$. The reaction was stopped by addition of $500 \mu \mathrm{l}$ of $20 \%$ trichloroacetic acid solution (Sigma-Aldrich Co.) with further centrifu- 
gation at $1000 \times \boldsymbol{g}$ for $7 \mathrm{~min}$. The supernatant was poured out and the pellet was washed twice with $1 \mathrm{ml}$ and then with $2 \mathrm{ml}$ of $1: 1(\mathrm{v} / \mathrm{v})$ ethanol/ethyl diacetate (POCh S.A., Sigma-Aldrich Co.). Each washing step was followed by centrifugation at $1000 \times \boldsymbol{g}$ for 7 min and disposal of the supernatant. After the final centrifugation the pellet was suspended in a solution of $6 \mu \mathrm{M}$ guanidine (SigmaAldrich Co.), $\mathrm{pH} 6.5$ and then incubated at $50^{\circ} \mathrm{C}$ with continuous shaking till the pellet became dissolved. The colorimetry was performed at the wavelength of $360 \mathrm{~nm}$ and the carbonyl group level was quantified per $1 \mathrm{mg}$ of protein versus the respective control sample.

Sulfhydryl group assessment. This was performed as described by Lou et al. [24]. Three hundred $\mu \mathrm{l}$ of the $10 \%$ homogenate was mixed with $600 \mu \mathrm{l}$ of sodium dodecyl sulphate (SDS; Sigma-Aldrich Co.), $1800 \mu \mathrm{l}$ of $10 \mathrm{mM}$ sodium-phosphate buffer, $\mathrm{pH}$ 8.0, and $300 \mu \mathrm{l}$ of $0.4 \mathrm{mM}$ solution of 5,5'-dithio-bis(2-nitrobenzoic acid) (DTNB or Ellman's Reagent; Sigma-Aldrich Co.) in sodium-phosphate buffer. After $15 \mathrm{~min}$ of incubation at room temperature the colorimetry was performed at the wavelength of $412 \mathrm{~nm}$. The sulfhydryl group level was quantified per $1 \mathrm{mg}$ of protein versus the control sample $(600 \mu$ l SDS +2100 $\mu \mathrm{l}$ of sodium-phosphate buffer $+300 \mu \mathrm{l}$ DTNB).

\section{Lipid hydroxyperoxide assessment.} Lipid hydroperoxides ( $\mathrm{LOOH})$ in samples of Syrian hamster kidneys were determined according to Thomas \& Poznansky [25]. This high sensitive method permits to measure lipid peroxides at level as low as 2 nmol per 1 $\mathrm{mg}$ of protein. The assay mixture contained $50 \mathrm{mM}$ sodium acetate ( $\mathrm{pH} 4$ for lipid peroxides and $\mathrm{pH} 4.5$ for standard cumene hydroperoxide), $0.1 \%$ Triton X-100, $100 \mu \mathrm{l}$ of 1.6 $\mathrm{mM} N, N, N^{\prime}, N^{\prime}$-tetramethylbenzidine in dimethylformamide, hemoglobin (100 $\mu \mathrm{g}$ for lipid peroxides and $300 \mu \mathrm{g}$ for cumene hydroperoxide) and $5-50 \mathrm{nmol}$ of peroxide in a total volume of $2 \mathrm{ml}$. The reaction was started by adding pure hemoglobin and the tubes were immediately transferred to an ice bath, kept closed with a lid in order to protect lipid peroxides from light. After 5 min of incubation in the ice bath, tubes were taken one at a time, $100 \mu \mathrm{l}$ of $1 \mathrm{M} \mathrm{HCl}$ was added and mixed and absorbance at $466 \mathrm{~nm}$ was measured immediately against a blank.

Electron paramagnetic resonance spectroscopy and spin labeling techniques. Characteristics of microsomal membrane polarity can be examined by 5-doxylstearic acid (5-DSA). 5-DSA dissolved in chloroform was evaporated under argon. Freshly prepared microsomal fractions (10 $\mathrm{mg}$ of protein) were incubated in spin label-coated tubes with gentle agitation for $30 \mathrm{~min}$ at $20^{\circ} \mathrm{C}$. Microsomal membrane lipid peroxidation was assessed by EPR spectroscopy using a paramagnetic reporter group incorporated into membranes which give a characteristic spectrum upon excitation with microwaves in a magnetic field. In brief, the increased production of reactive oxygen species can result in a significant accumulation of more polar hydroperoxides. The amphipathic spin probe 5-DSA is thought to intercalate within both halves of a lipid bilayer so that its long alkyl chain is roughly parallel to the alkyl chain of membrane lipids, and its paramagnetic nitroxide moiety near the lipid-water interface [26]. Rapid anisotropic motion occurs about the long axis of the spin probe providing opportunity to detect polarity changes at the relevant EPR parameter of +1 low-field line. A reliable parameter of the environment of 5-DSA calculated from direct measurements of the parallel and perpendicular components of the hyperfine tensor of the spin label, is the isotropic splitting factor $\mathrm{a}_{0}$. A high $a_{0}$ reflects increased polarity of the microsomal membrane.

Statistical analysis. Each assessment was performed three times and as the data were not significantly different, the results were combined. The analysis was performed using ANOVA models, Student's $t$ test and $r$-Pearson correlation test. 
The analysis was made using statistical software SPSS/93, thanks to generous assistance of Prof. B. Wojciszke (University of Gdańsk, Poland).

\section{RESULTS}

Oxidative stress to proteins, measured by the content of the carbonyl and sulfhydryl groups per $1 \mathrm{mg}$ of total protein, appeared higher in the estradiol receiving group of hamsters than in control group (Table 1). The difference was statistically significant.

In detail, the levels of carbonyls in subcellular fractions of the control group were similar and did not exceed $3 \mathrm{nmol} / \mathrm{mg}$ of pro-
The levels of sulfhydryl groups showed a reciprocal correlation in comparison with those of carbonyls. Their level in fractions of the estrogen receiving group was lower both in mitochondrial and microsomal fractions, the latter difference being more significant. Statistical analysis displayed a significant decrease in the sulfhydryl group content in fractions of the estrogen receiving group as compared with the respective fractions of the control group. The decrease was most significant in the microsomal fraction (Table 3).

In control group of hamsters LOOH level was not different from that detected in estrogen treated hamsters (Table 1). No significant EPR spectral changes of 5-DSA in microsomal membranes were visible after estradiol treat-

Table 1. Levels of carbonyl and sulfhydryl groups and lipid hydroperoxide (LOOH) in postnuclear supernatants of kidney tissue of male Syrian hamsters treated with estradiol for one month and in untreated control group (statistical significance in Student's $t$ tests)

\begin{tabular}{llll}
\hline \multirow{2}{*}{ Group } & $\begin{array}{l}\text { Carbonyl groups } \\
\text { nmol/mg of protein }\end{array}$ & Sulfhydryl groups & LOOH \\
& 4.43 & 69.73 & 40 \\
\hline Group 1 - Estradiol & 2.31 & 101.52 & 39.5 \\
Group 2 - Control & $P<0.005$ & $P<0.05$ & not significant \\
\hline Statistical significance & & & \\
\hline
\end{tabular}

tein. The carbonyl levels in the fractions of the estrogen-receiving group were significantly higher as compared with the respective fractions of the control group. The most significant increase was observed in the microsomal fraction (Table 2). ment as compared to respective control (Fig. 1), which is in accordance with a parallel lack of accumulation of lipid hydroperoxides.

The correlation between carbonyl and sulfhydryl group levels in the respective subcellular fractions of either group displayed

Table 2. Carbonyl group levels in subcellular fractions of kidneys of male Syrian hamsters.

Statistics (measured by Student's $t$ test): in rows - within each group between subcellular fractions, in columns - between groups.

\begin{tabular}{llll}
\hline \multirow{2}{*}{ Group } & \multicolumn{2}{l}{ Subcellular fraction } & \multirow{2}{*}{ Statistical significance } \\
\cline { 2 - 3 } & mitochondria $\quad$ microsomes & \\
\cline { 2 - 3 } & carbonyl groups $/ \mathrm{mg}$ of protein & \\
\hline Group 1 - Estradiol & 2.86 & 13.77 & not significant \\
Group 2 - Control & 1.97 & 2.14 & \\
\hline Statistical significance & not significant & $P<0.001$ & \\
\hline
\end{tabular}


Table 3. Sulfhydryl group levels in subcellular fractions of kidneys of male Syrian hamsters.

Statistics (measured by Student's $t$ test): in rows - within each group between subcellular fractions, in columns - between groups.

\begin{tabular}{llll}
\hline \multirow{2}{*}{ Group } & \multicolumn{2}{l}{ Subcellular fraction } & \multirow{2}{*}{ Statistical significance } \\
\cline { 2 - 3 } & \multicolumn{2}{l}{ mitochondria $\quad$ microsomes } & \\
\cline { 2 - 3 } sulfhydryl groups $/ \mathrm{mg}$ of protein & & \multicolumn{2}{l}{ not significant } \\
\hline Group 1 - Estradiol & 54.67 & 23.54 & \\
Group 2 - Control & 84.67 & 96.68 & $P<0.005$ \\
\hline Statistical significance & $P<0.05$ & &
\end{tabular}

a similar $r$-Pearson correlation factor for the estrogenized group $r=-0.73$; for the control group $r=-0.67$. Both correlations were statistically significant at the level of $P<0.05$. The

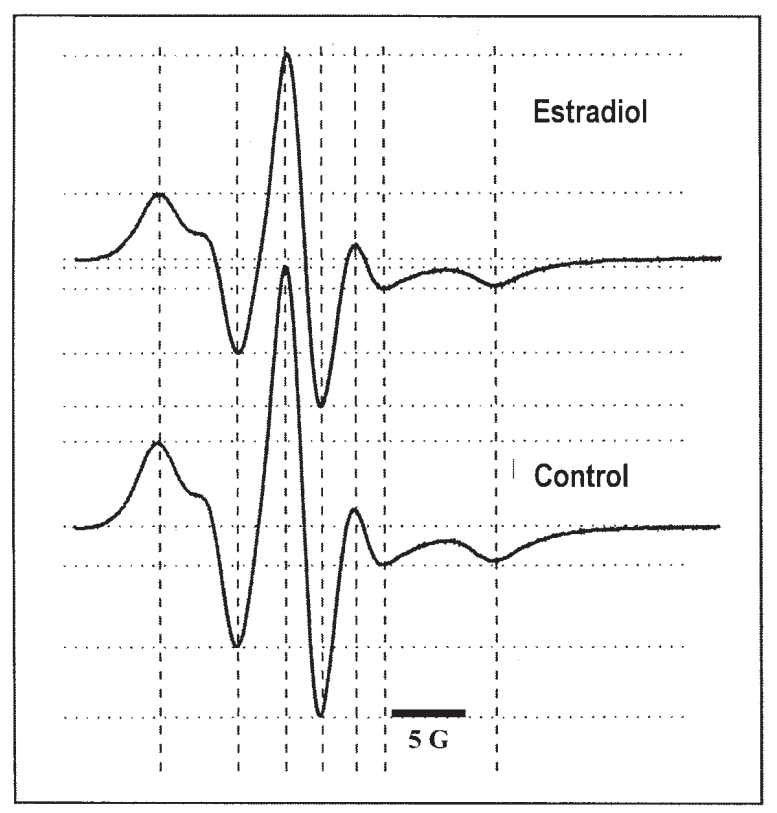

Figure 1. EPR spectra of 5-doxylstearic acid in microsomal fraction from kidneys of estradioltreated male hamsters and control animals

values of $r$-Pearson correlation between the level of carbonyl and sulfhydryl groups and the level of the hydroperoxide were calculated as 0.12 and 0.07 , respectively, and were not statistically significant.

\section{DISCUSSION}

Chronic exposure of male Syrian hamsters to estradiol $\left(\mathrm{E}_{2}\right)$ or other estrogens (e.g. diethylstilbestrol) results in induction of estrogen dependent kidney neoplasm that is observed as a macroscopic tumor. The incidence of tumor occurrence in estrogenized animals reaches a $100 \%$ of cases within a period of 9 to 12 months [8, 9, 27]. The cancer is estrogen dependent regarding both its induction and growth - it is found to spontaneously differentiate back to normal kidney tissue after estrogenization has been ceased [28-29].

Although this neoplasm has been widely described in the literature, its pathogenesis still remains unclear, and nevertheless, many authors suspect free radical-mediated cancer induction.

Liehr and coworkers [30-33] reported a decrease in quinone reductase and catalase activities and increase in glutathione peroxidase I and II activities in kidney of male Syrian hamsters treated with $\mathrm{E}_{2}$ for one month. In the same study they described an increase in microsomal cytochrome $\mathrm{P} 450$ content in animals treated with $\mathrm{E}_{2}$ for the same time. They did not manage to show a significant increase in peroxidative activity of that cytochrome, which may suggest that the oxidative stress is targeted specifically at certain biomolecules and does not affect all the microsomal fraction particles. Although many authors suggest that protein peroxidation may be preceded by 
lipid peroxidation, there is also strong evidence supporting the hypothesis that the oxidation pattern of proteins may be different from that of lipids [34]. Schnurr et al. [35] have proposed that the reaction of mammalian 15-lipoxygenases with submitochondrial particles displays the peculiarity of a high oxygen consumption that is not due to oxidative conversion of polyenoic fatty acids or other lipids, but due to oxidative breakdown of amino-acid residues of the membrane proteins. Lipoxygenase-induced oxidative modification of mitochondria membrane proteins has been found to be coenzyme Q-mediated [35]. Such a finding points to a possible specific interaction of semiquinone radical with membrane proteins. A significant oxidative stress affecting proteins measured by changes of carbonyl and sulfhydryl group levels proves that even at an early stage of carcinogenesis the kidney proteins are susceptible to immense free radical-induced oxidative damage. We also noticed a significant increase in carbonyl group level in hamster kidneys after 5-hour exposition to estradiol: 4.23 vs. 1.76 for the control $(P<0.05)$ (results not shown). It is interesting that such changes persisted after one month of treatment, i.e. close to the time-point when the first morphological changes become evident. Our histopathological results suggest that the first preneoplastic lesion cannot be seen earlier than 4 weeks after the initial estradiol implantation (results not shown). Moreover, Hou et al. [36] reported only transient protooncogene expression (c-myc, c-fos, c-jun) in hamsters after initial exposition to estradiol for 2 and $5 \mathrm{~h}$. On the other hand, prolonged exposition (1 to 5 months) resulted in significant and stable elevation of protooncogene expression in hamster kidney [36]. The localization of the stress seems to support the hypothesis that estradiol-4-hydroxylase is the key enzyme involved in the process of carcinogenesis. The enzyme producing large amounts of 4-hydroxyestradiol provides the substrate for oxidoreductive cycling catalyzed by the increased amount of cytochrome P450 [37, 38]. This results in extensive induction of semiquinones and quinones, which can damage DNA as well as proteins. In conclusion, our results suggest that kidney endoplasmic reticulum membrane proteins are the early target biomolecules at the inductive stage of estrogen-induced model of nephrocarcinogenesis.

\section{R E F E R E N C ES}

1. Kirkman, H. (1959) Estrogen-induced tumors of the kidney. III. Growth characteristics in the Syrian hamster. Nat. Cancer Inst. Monogr. 1, 1-57.

2. Liehr, J.G., Fang, W.F., Sirbasku, D.A. \& Ari-Ulubelen, A. (1986) Carcinogenicity of catechol estrogens in Syrian hamsters. J. Steroid Biochem. 24, 353-356.

3. Bhat, H.K., Hacker, H.J., Bannasch, P., Thompson, E.A. \& Liehr, J.G. (1993) Localization of estrogen receptors in interstitial cells of hamster kidney and in estradiol-induced renal tumors as evidence of the mesenchymal origin of this neoplasm. Cancer Res. 53, 5447-5451.

4. Liehr, J.G. (1998) Catecholestrogens in the induction of tumors in the kidney of the Syrian hamster. Adv. Pharmacol. 42, 824-828.

5. Sirbasku, D.A. \& Moreno-Cuevas, J.E. (2000) Estrogen mitogenic action. II. Negative regulation of the steroid hormone-responsive growth of cell lines derived from human and rodent target tissue tumors and conceptual implications. In vitro Cell Dev. Biol. Anim. 36, 428-446.

6. Hacker, H.J., Bannasch, P. \& Liehr, J.G. (1988) Histochemical analysis of the development of estradiol-induced kidney tumors in male Syrian hamsters. Cancer Res. 48, 971-976.

7. Yager, J.D. (2000) Endogenous estrogens as carcinogens through metabolic activation. $J$. Natl. Cancer Inst. Monogr. 27, 67-73. 
8. Oberley, T.D., Gonzalez, A., Lauchner, L.J., Oberley, L.W. \& Li, J.J. (1991) Characterization of early kidney lesions in estrogen-induced tumors in the Syrian hamsters. Cancer Res. 51, 1922-1929.

9. Goldfarb, S. \& Pugh, T.D. (1990) Morphology and anatomic localization of renal microneoplasms and proximal tubule dysplasias induced by four different estrogens in the hamsters. Cancer Res. 50, 113-119.

10. Roy, D., Kalyanaraman, B. \& Liehr, J.G. (1991) Xanthine oxidase-catalyzed reduction of estrogen quinones to semiquinones and hydroquinones. Biochem. Pharmacol. 42, 1627-1631.

11. Liehr, J.G. (2000) Is estradiol a genotoxic mutagenic carcinogen? Endocr. Rev. 21, 40-54.

12.Li, J.J. \& Li, S.A. (1987) Estrogen carcinogenesis in Syrian hamster tissue: Role of metabolism. Fed. Proc. 46, 1858-1863.

13. Stalford, A.C., Maggs, J.L., Gilchrist, T.L. \& Park, B.K. (1994) Catecholestrogens as mediators of carcinogenesis: Correlation of aromatic hydroxylation of estradiol and its fluorinated analogs with tumor induction in Syrian hamsters. Mol. Pharmacol. 45, 1259-1267.

14. Li, S.A., Klicka, J.K. \& Li, J.J. (1985) Estrogen 2- and 4-hydroxylase activity, catechol estrogen formation, and implications for estrogen carcinogenesis in the hamster kidney. Cancer Res. 45, 181-185.

15. Weisz, J., Bui, Q.D., Roy, D. \& Liehr, J.G. (1992) Elevated 4-hydroxylation of estradiol by hamster kidney microsomes: A potential pathway of metabolic activation of estrogens. Endocrinology 131, 655-661.

16. Ashburn, S.P., Han, X. \& Liehr, J.G. (1993) Microsomal hydroxylation of 2- and 4-fluoroestradiol to catechol metabolites and their conversion to methyl ethers: Catechol estrogens as possible mediators of hormonal carcinogenesis. Mol. Pharmacol. 43, 534-541.
17. Liehr, J.G. \& Ricci, M.J. (1996) 4-Hydroxylation of estrogens as marker of human mammary tumors. Proc. Natl. Acad. Sci. U.S.A. 93, 3294-3296.

18. Liehr, J.G., Ricci, M.J., Jefcoate, C.R., Hannigan, E.V., Hokanson, J.A. \& Zhu, B.T. (1995) 4-Hydroxylation of estradiol by human uterine myometrium and myoma microsomes: Implications for the mechanism of uterine tumorigenesis. Proc. Natl. Acad. Sci. U.S.A. 92, 9220-9224.

19. Hammond, D.K., Zhu, B.T., Wang, M.Y., Ricci, M.J. \& Liehr, J.G. (1997) Cytochrome P450 metabolism of estradiol in hamster liver and kidney. Toxicol. Appl. Pharmacol. 145, 54-60.

20.Zhu, B.T. \& Conney, A.H. (1998) Functional role of estrogens metabolism in target cells: Review and perspectives. Carcinogenesis 19, $1-27$.

21. Li, J.J., Li, S.A., Oberley, T.D. \& Parsons, J.A. (1995) Carcinogenic activities of various steroidal and nonsteroidal estrogens in the hamster kidney: Relation to hormonal activity and cell proliferation. Cancer Res. 55, 4347-4351.

22.Li, J.J., Gonzales, A., Banerjee, S., Banerjee, S.K. \& Li, S.A. (1993) Estrogen carcinogenesis in the hamster kidney: Role of cytotoxicity and cell proliferation. Environ. Health Perspect. 101 (Suppl. 5), 259-264.

23. Oliver, C.N., Ahn, B.W., Moerman, E.J., Goldstein, S. \& Stadtman, E.R. (1987) Age-related changes in oxidized proteins. J. Biol. Chem. 262, 5488-5491.

24. Lou, M.F., Poulsen, L.L. \& Zeigler, D.M. (1987) Cellular protein-mixed disulfides. Methods Enzymol. 143, 124-129.

25. Thomas, P.D. \& Poznansky, M.J. (1990) A modified tetramethylbenzidine method for measuring lipid hydroperoxides. Anal. Biochem. 188, 228-232. 
26. Butterfield, D.A. (1982) Spin labeling in disease. Biol. Magn. Reson. 4, 1-78.

27. Li, J.J. \& Li, S.A. (1984) Estrogen-induced tumorigenesis in hamsters: Roles for hormonal and carcinogenic activities. Arch. Toxicol. 55, 110-118.

28. Sirbasku, D.A. \& Kirkland, W.L. (1976) Control of cell growth. IV. Growth properties of a new cell line established from an estrogen-dependent kidney tumor of the Syrian hamster. Endocrinology 98, 1260-1272.

29. Roy, D. \& Liehr, J.G. (1990) Inhibition of estrogen-induced kidney carcinogenesis in Syrian hamsters by modulators of estrogen metabolism. Carcinogenesis 11, 567-570.

30. Roy, D. \& Liehr, J.G. (1988) Temporary decrease in renal quinone reductase activity induced by chronic administration of estradiol to male Syrian hamsters. Increased superoxide formation by redox cycling of estrogen. J. Biol. Chem. 263, 3646-3651.

31. Liehr, J.G., Hall, E.R., Avitts, T.A., Randerath, E. \& Randerath, K. (1987) Localization of estrogen-induced DNA adducts and cytochrome P- 450 activity at the site of renal carcinogenesis in the hamster kidney. Cancer Res. 47, 2156-2159.

32. Roy, D. \& Liehr, J.G. (1990) Inhibition of estrogen-induced kidney carcinogenesis in Syrian hamsters by modulators of estrogen metabolism. Carcinogenesis 11, 567-570.

33.Liehr, J.G., Roy, D., Ari-Ulubelen, A., Bui, Q.D., Weisz, J. \& Strobel, H.W. (1991) Effect of chronic estrogen treatment of Syrian ham- sters on microsomal enzymes mediating formation of catecholestrogens and their redox cycling: Implications for carcinogenesis. $J$. Steroid Biochem. 35, 555-560.

34. Antosiewicz, J., Popinigis, J., Wozniak, M., Damiani, E., Carloni, P. \& Greci, L. (1995) Effects of indolinic and quinolinic aminoxyls on protein and lipid peroxidation of rat liver microsomes. Free Radical Biol. Med. 18, 913-917.

35. Schnurr, K., Hellwing, M., Seidemann, B., Jungblut, P., Kuhn, H., Rapoport, S.M. \& Schewe, T. (1996) Oxygenation of biomembranes by mammalian lipoxygenases: The role of ubiquinone. Free Radical Biol. Med. 20, 11-21.

36. Hou, X., Li, J.J., Chen, W. \& Li, S.A. (1996) Estrogen-induced proto-oncogene and suppressor gene expression in the hamster kidney: Significance for estrogen carcinogenesis. Cancer Res. 56, 2616-2620.

37. Wang, M.Y. \& Liehr, J.G. (1995) Induction by estrogens of lipid peroxidation and lipid peroxide-derived malonaldehyde-DNA adducts in male Syrian hamsters: Role of lipid peroxidation in estrogen-induced kidney carcinogenesis. Carcinogenesis 16, 1941-1945.

38.Liehr, J.G., Roy, D., Ari-Ulubelen, A., Bui, Q.D., Weisz, J. \& Strobel, H.W. (1990) Effect of chronic estrogen treatment of Syrian hamsters on microsomal enzymes mediating formation of catecholestrogens and their redox cycling: Implications for carcinogenesis. $J$. Steroid Biochem. 35, 555-560. 